\title{
Static Heat Energy Balance Mathematical Model for an Iron Blast Furnace
}

\author{
Ayush Bhattacharya ${ }^{1,}$, , Sadhasivam Muthusamy ${ }^{2}$ \\ ${ }^{1}$ School of Mechanical Engineering, VIT University, Vellore, India \\ ${ }^{2}$ Blast Furnace Department, JSW Steel Ltd., Salem, India \\ Email address: \\ ayush.bhattacharya2015@gmail.com (A. Bhattacharya) \\ ${ }^{*}$ Corresponding author
}

\section{To cite this article:}

Ayush Bhattacharya, Sadhasivam Muthusamy. Static Heat Energy Balance Mathematical Model for an Iron Blast Furnace. International Journal of Mineral Processing and Extractive Metallurgy. Vol. 2, No. 5, 2017, pp. 57-67. doi: 10.11648/j.ijmpem.20170205.11

Received: August 29, 2017; Accepted: September 11, 2017; Published: October 10, 2017

\begin{abstract}
In this study a static heat energy balance analysis has been carried out for an iron blast furnace. The objective of this work is to provide a mathematical calculation model of the heat distributions for the various components of the blast furnace. The model presented, is also indicative to the amount of excess fuel being charged. To prepare a proper heat balance, the first step is to attain a proper mass balance calculation. To do so, each input and output materials has been analysed, and the respective elemental compositions have been calculated. All major components and reactions of a blast furnace have been included in the study. Each calculation has been done with sufficient details, to allow estimation of heat requirements, according to the working conditions of a blast furnace.
\end{abstract}

Keywords: Blast Furnace, Heat Energy Balance, Blast Furnace Reactions, Blast Furnace Efficiency

\section{Introduction}

Heat energy analysis is a very major study for the proper operation of a blast furnace. Heat balance is an account of the input and output of heat in a process, which follows the first law of thermodynamics. A proper heat balance not only helps to predict the efficiency of a furnace, but to also eliminate any excessive fuel wastages. Reduced fuel requirement not only reduces production costs, but more importantly saves a portion of our rapidly depleting natural resources. To get a proper heat balance, a proper material balance is a necessity. A material balance is simply an account of input and output of mass, governed by the law of conservation of mass. A proper material balance will provide accurate quantitative values, thereby simplifying calculations in each step of the heat balance. Besides this, a detailed study of each input and output components of the blast furnace has to be carried out, which includes: 1. Fuel Supplied; 2. Combustion Air or Hot Blast; 3. Blast Furnace Reactions; 4. Hot Metal; 5. Volatile Matter in Input Materials; 6. Moisture in Input Materials; 7. Blast Furnace Gas; 8. Dust Collected; 9. Blast Furnace Slag; 10. Cooling Water Supplied; 11. Other Heat Losses (Heat Loss through the tuyeres, Conduction, Convection, Radiation etc.).
A heat balance analysis for a continuous production blast furnace was presented by [1]. The authors gave a simplified model to calculate heat distributions for various components of the furnace. However, the authors did not provide any insight to the reactions occurring in the furnace. Also, no sub-divisions to the calculations were shown. Taking [1] as reference, the following study has been conducted to calculate exact heat distributions of each input and output component of the furnace, in detail. Various standard textbooks [2-5], and papers [11-14] have been referred to get an in-depth insight into the factors which should be taken into consideration, and the reactions taking place in the different regions of the furnace. The standard enthalpy of formation of compounds data has been taken from [6], the enthalpy data of elements and compounds at high temperatures has been taken from [7] and the heat of solution data has been taken from [8]. Composition analysis of all materials has been carried out using XRF analysis, and BF gas analysis has been carried out using Orsat absorption method.

\section{Working of a Blast Furnace}

A blast furnace is a huge, steel rack lined with refractory 
bricks, which is used to convert iron oxide into pig iron. The blast furnace is an example of a counter current reactor where solids descend and gasses ascend. The fuel (coke, coal, nut coke) and other raw materials (iron ore, sinter, dunite, dolomite, quartzite) are weighed, and charged into the furnace from the top.

Coke is a mixture of coals, crushed and then heated to remove most of the volatile matter. Coke has higher calorific values due to the presence of more carbon content. Nut coke is smaller in size and has a lesser calorific value than coke. Sinter is produced by agglomerating iron ore fines with other plant wastages, having some iron percentages, such as screened sinter fines, flue dust and sludge from the blast furnace, and scales from mills. Limestone and dolomite are added to maintain the required basicity of sinter. Coke fines are used in the coke oven plant, to heat the iron ore fines along with the flux material, to form lumps of sinter. The iron ore is found in mainly two forms, Hematite $\left(\mathrm{Fe}_{2} \mathrm{O}_{3}\right)$ and Magnetite $\left(\mathrm{Fe}_{3} \mathrm{O}_{4}\right)$.

Air is collected from the atmosphere and heated to $1200^{\circ} \mathrm{C}$ with the help of giant hot blast stoves. From the stoves the hot combustion air is given sufficient oxygen enrichment, and supplied into the furnace at high pressure, through the tuyeres, as hot blast. The coke descends to the bottom of the furnace and is ignited by the hot combustion air blast. The coke reacts to produce $\mathrm{CO}_{2}$ and heat, raising temperatures of the combustion region to around $1900^{\circ} \mathrm{C}$. The wustite ore, having a high melting point, melts in this region. The $\mathrm{CO}_{2}$ produced again reacts with excess $\mathrm{C}$, to produce $\mathrm{CO}$ gas. This hot gas then moves upwards in the furnace reducing the iron oxides and the other input materials. Other than reduction by $\mathrm{CO}$ gas (Indirect Reduction), other reducing processes are reduction by $\mathrm{C}$ (Direct Reduction) and reduction by $\mathrm{H}_{2}$. Dunite, dolomite and quartzite are slag producing agents, which produce slag along with iron ore impurities such as alumina and silica, and help in the removal of elements like sulphur and phosphorus. The raw materials require around 6 to 8 hours to descend to the bottom of the furnace, as the final products: hot metal and slag. The hot metal and slag flow out of the blast furnace through the tapping hole. The hot metal flows through the runner and is collected in the ladles. The slag having a lesser density than that of the hot metal, separates out from the hot metal, as the slag runner is placed in a different direction. The slag is granulated by sprinkling water over it and later sent to cement factories, where it used as a raw material. During reactions, various other gases are produced at various levels inside the furnace. These gases leave the furnace and is known as Blast Furnace Gas (BF Gas). The BF gas carries small particulate matter (dust), which are removed by passing the gas through the Dust Catcher and then the Gas Cleaning Plant (GCP). The BF Gas carries significant amount of energy, and after removal of dust, is reused in various areas of the plant. The dust collected in the Dust Catcher and GCP is used as a raw material for sinter. Figure 1 shows a schematic diagram of a blast furnace plant.

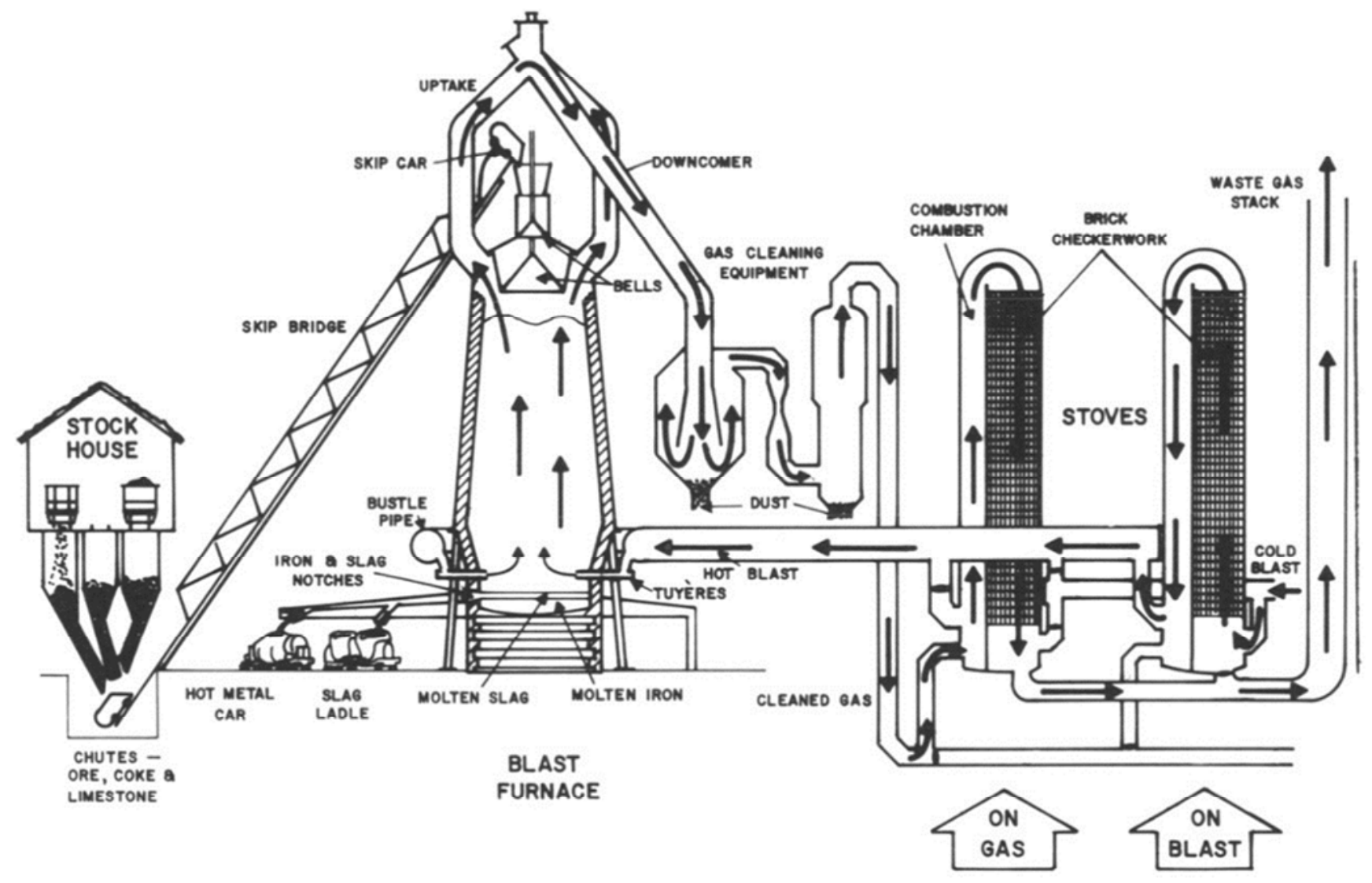

Figure 1. Schematic Diagram of Blast Furnace Plant [2]. 


\section{Heat Balance Calculations}

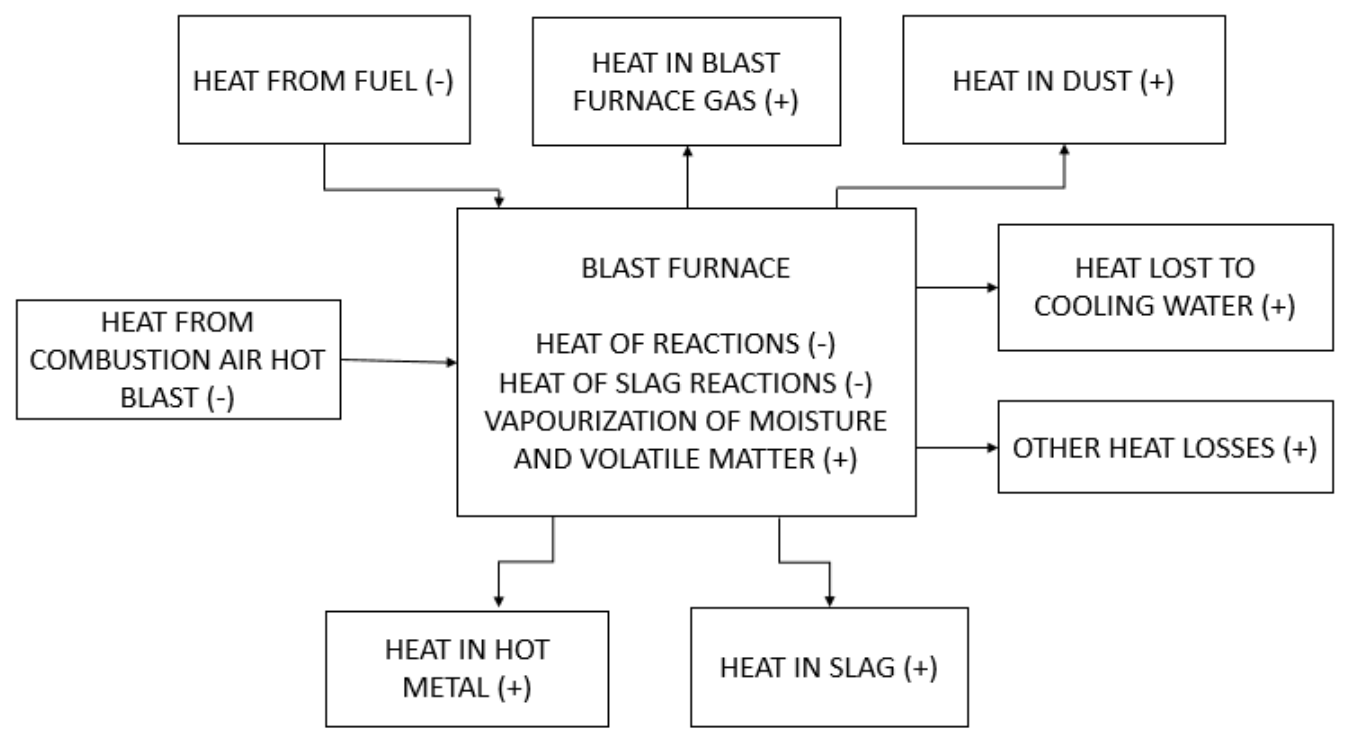

Figure 2. Heat Energy Flow Diagram.

All data for the study has been taken from the month of April 2017. A heat energy flow diagram is shown in Figure 2 for a demonstration of the calculations. (-) sign indicates components which release heat, whereas $(+)$ sign indicates heat absorbing components.

Hot Metal Production in April 2017=48411 Tons

\subsection{Fuel Analysis}

The calorific values of coke, coal and nut coke are tested for each batch, on arrival. These fuel analysis values have been taken for the fuel used in the month of April, 2017.

Quantity of Coke Consumed $=381 \mathrm{Kg} / \mathrm{THM}$

Calorific Value of Coke $=7020 \mathrm{Kcal} / \mathrm{Kg}$

Quantity of Coal Consumed= $130 \mathrm{Kg} / \mathrm{THM}$

Calorific Value of Coal $=5605 \mathrm{Kcal} / \mathrm{Kg}$

Quantity of Nut Coke Consumed=19 Kg/THM

Calorific Value of Nut Coke $=6850 \mathrm{Kcal} / \mathrm{Kg}$

$$
\begin{aligned}
& \text { Energy Generated from Coke Combustion }(\mathrm{A} 1)=381 \mathrm{Kg} / \mathrm{THM} * 7020 \mathrm{Kcal} / \mathrm{Kg} \\
& =2674620 \mathrm{Kcal} / \mathrm{THM} \\
& =728650 \mathrm{Kcal} / \mathrm{THM} \\
& =130150 \mathrm{Kcal} / \mathrm{THM}
\end{aligned}
$$

Total Energy Generated from Fuel Input $=\mathrm{A} 1+\mathrm{A} 2+\mathrm{A} 3$

$$
=3533420 \mathrm{Kcal} / \mathrm{THM}(-)
$$

\subsection{Combustion Air or Hot Blast Analysis}

Volume of Moist Blast $=1181 \mathrm{~m}^{3} / \mathrm{THM}$

It is taken that atmospheric air contains $21 \%$ Oxygen $\left(\mathrm{O}_{2}\right)$ and $79 \%$ Nitrogen $\left(\mathrm{N}_{2}\right)$. Also, 15 gm moisture is considered to be present per $\mathrm{m}^{3}$ of atmospheric air.

Quantity of $\mathrm{H}_{2} \mathrm{O}$ in Moist Blast $=15 \mathrm{gm} / \mathrm{m}^{3 *} 1181 \mathrm{~m}^{3} / \mathrm{THM}$

$$
\begin{aligned}
& =17715 \mathrm{gm} / \mathrm{THM} \\
& =984 \mathrm{moles} / \mathrm{THM} \\
& =21 \mathrm{~m}^{3} / \mathrm{THM}
\end{aligned}
$$

Volume of Dry Blast $=1181 \mathrm{~m}^{3} / \mathrm{THM}-21 \mathrm{~m}^{3} / \mathrm{THM}$

$$
=1160 \mathrm{~m}^{3} / \mathrm{THM}
$$

Steam is supplied to the combustion air at $21 \mathrm{gm} / \mathrm{m}^{3}$.

Total Volume of $\mathrm{H}_{2} \mathrm{O}$ in combustion air $=21 \mathrm{gm} / \mathrm{m}^{3 *} 1181 \mathrm{~m}^{3} / \mathrm{THM}+17715 \mathrm{gm} / \mathrm{THM}$

$$
\begin{aligned}
& =42516 \mathrm{gm} / \mathrm{THM} \\
& =2362 \mathrm{moles} / \mathrm{THM}
\end{aligned}
$$

Oxygen Enrichment of $2.39 \%$ is given to the combustion air. 
Volume of $\mathrm{O}_{2}$ Enrichment given $=0.0239 * 1181 \mathrm{~m}^{3} / \mathrm{THM}$

$$
=28 \mathrm{~m}^{3} / \mathrm{THM}
$$

Total Volume of $\mathrm{O}_{2}$ in combustion air $=244 \mathrm{~m}^{3} / \mathrm{THM}+28 \mathrm{~m}^{3} / \mathrm{THM}$

$$
\begin{aligned}
& =272 \mathrm{~m}^{3} / \mathrm{THM} \\
& =388576 \mathrm{gm} / \mathrm{THM} \\
& =12143 \mathrm{moles} / \mathrm{THM}
\end{aligned}
$$

Total Volume of $\mathrm{N}_{2}$ in combustion air $=916 \mathrm{Nm}^{3} / \mathrm{THM}$

$$
\begin{aligned}
& =1145508 \mathrm{gm} / \mathrm{THM} \\
& =40911 \mathrm{moles} / \mathrm{THM}
\end{aligned}
$$

The complete combustion air composition results are shown in Table 1.

Table 1. Combustion Air Composition Analysis.

\begin{tabular}{cccc}
\hline Element & $\mathbf{H}_{\mathbf{2}} \mathbf{O}$ & $\mathbf{O}_{\mathbf{2}}$ & $\mathbf{N}_{\mathbf{2}}$ \\
\hline $\mathrm{m}^{3} / \mathrm{THM}$ & 53 & 272 & 916 \\
Moles/THM & 2362 & 12143 & 40911 \\
\hline
\end{tabular}

$$
\begin{aligned}
& \text { Latent Heat of } \mathrm{H}_{2} \mathrm{O}(\text { Liquid } \rightarrow \text { Gas })(\mathrm{B} 1)=42.5 \mathrm{Kg} / \mathrm{THM} * 540 \mathrm{Kcal} / \mathrm{Kg} \\
& =22950 \mathrm{Kcal} / \mathrm{THM}
\end{aligned}
$$

Sensible Heat of $\mathrm{O}_{2}(\mathrm{~B} 3)=12143 \mathrm{moles} / \mathrm{THM}^{*} 9.37 \mathrm{Kcal} / \mathrm{moles}$

$$
=113780 \mathrm{Kcal} / \mathrm{THM}
$$

Sensible Heat of $\mathrm{N}_{2}(\mathrm{~B} 4)=40911 \mathrm{moles} / \mathrm{THM} * 8.93 \mathrm{Kcal} / \mathrm{moles}$

$$
=365335 \mathrm{Kcal} / \mathrm{THM}
$$

Total Heat of all combustion air constituents $=\mathrm{B} 1+\mathrm{B} 2+\mathrm{B} 3+\mathrm{B} 4$

$$
=528378 \mathrm{Kcal} / \mathrm{THM}(-)
$$

\begin{tabular}{|c|c|c|c|c|c|c|c|}
\hline Element & $\mathrm{C}$ & $\mathrm{Fe}_{2} \mathrm{O}_{3}$ & $\mathrm{SiO}_{2}$ & $\mathbf{P}_{2} \mathbf{O}_{5}$ & MnO & $\mathrm{TiO}_{2}$ & $\mathbf{A l}_{2} \mathbf{O}_{3}$ \\
\hline$\%$ & 3.35 & 89.4 & 3.57 & 0.025 & 0.55 & 0.16 & 2.24 \\
\hline $\mathrm{Kg} / \mathrm{THM}$ & 19.45 & 509.11 & 14.73 & 0.66 & 2.62 & 0.93 & 13.01 \\
\hline Moles/THM & 1621.45 & 3182 & 245.58 & 4.68 & 37 & 11.62 & 127.55 \\
\hline
\end{tabular}

\subsection{Composition Analysis}

For simplicity in calculations, it has been assumed the input iron ore to be only in the form of $\mathrm{Hematite}\left(\mathrm{Fe}_{2} \mathrm{O}_{3}\right)$. The percentage compositions of the various input materials are studied using XRF analysis, which is carried out for every batch of input materials, on arrival. The values are of that input materials, used in the month of April 2017. Only the percentage

\begin{tabular}{|c|c|c|c|c|c|c|c|c|c|}
\hline Element & $\mathrm{Fe}_{2} \mathrm{O}_{3}$ & FeO & $\mathrm{SiO}_{2}$ & $\mathrm{CaO}$ & MgO & $\mathbf{P}_{2} \mathbf{O}_{5}$ & MnO & $\mathrm{TiO}_{2}$ & $\mathbf{A l}_{2} \mathbf{O}_{3}$ \\
\hline$\%$ & 66.28 & 10.23 & 3.57 & 11 & 2.26 & 0.037 & 0.35 & 0.16 & 2.65 \\
\hline $\mathrm{Kg} / \mathrm{THM}$ & 707.04 & 113.11 & 40.08 & 112.14 & 28.8 & 1.86 & 3.27 & 1.76 & 29.72 \\
\hline Moles/THM & 4419 & 1571 & 668 & 2002.5 & 720 & 13.09 & 46 & 22 & 291.42 \\
\hline
\end{tabular}
composition of the elements, taken into consideration for this study, has been shown.

\subsubsection{Iron Ore}

Total Quantity Used $=28118$ Tons

Moisture Content $=2.1 \%=603$ Tons $=12.46 \mathrm{Kg} / \mathrm{THM}=692 \mathrm{moles} / \mathrm{THM}$

Dry Weight $=27572$ Tons

The iron ore composition analysis results is shown in Table 2.

Table 2. Iron Ore Composition Analysis.

\subsubsection{Sinter}

Total Quantity Used= 53101 Tons

The sinter composition analysis results is shown in Table 3.

Table 3. Sinter Composition Analysis.

\subsubsection{Dunite}

Total Quantity Used $=1232$ Tons

Moisture Content $=2.5 \%=31$ Tons $=0.64 \mathrm{Kg} / \mathrm{THM}=35.35 \mathrm{moles} / \mathrm{THM}$ 
Dry Weight $=1201$ Tons

The dunite composition analysis results is shown in Table 4.

Table 4. Dunite Composition Analysis.

\begin{tabular}{|c|c|c|c|c|c|}
\hline Element & $\mathrm{Fe}_{2} \mathrm{O}_{3}$ & $\mathrm{CaCO}_{3}$ & $\mathrm{MgCO}_{3}$ & $\mathrm{SiO}_{2}$ & $\mathbf{A l}_{2} \mathbf{O}_{3}$ \\
\hline$\%$ & 4.5 & 0.88 & 44.36 & 36.25 & 0.98 \\
\hline $\mathrm{Kg} / \mathrm{THM}$ & 1.12 & 0.22 & 11.34 & 9 & 0.24 \\
\hline Moles/THM & 7 & 2.18 & 135 & 150 & 2.38 \\
\hline
\end{tabular}

\subsubsection{Dolomite}

Total Quantity Used $=324$ Tons

Moisture Content $=0 \%$

The dolomite composition analysis results is shown in Table 5.

Table 5. Dolomite Composition Analysis.

\begin{tabular}{|c|c|c|c|}
\hline Element & $\mathrm{CaCO}_{3}$ & $\mathrm{MgCO}_{3}$ & $\mathrm{SiO}_{2}$ \\
\hline$\%$ & 43.62 & 33.6 & 12.4 \\
\hline $\mathrm{Kg} / \mathrm{THM}$ & 2.92 & 2.25 & 0.83 \\
\hline Moles/THM & 29.2 & 26.85 & 13.77 \\
\hline
\end{tabular}

\subsubsection{Quartzite}

Total Quantity Used $=238$ Tons

Moisture Content $=2.5 \%=6$ Tons $=6.83$ Moles $/ \mathrm{THM}$

Dry Weight $=232$ Tons

The quartzite composition analysis results is shown in Table 6.

Table 6. Quartzite Composition Analysis.

\begin{tabular}{cccc}
\hline Element & $\mathbf{S i O}_{2}$ & $\mathbf{A l}_{\mathbf{2}} \mathbf{O}_{3}$ & \\
\hline$\%$ & 98 & \\
$\mathrm{Kg} / \mathrm{THM}$ & 4.68 & 0.3 \\
$\mathrm{Moles} / \mathrm{THM}$ & 78 & 0.01 \\
\end{tabular}

\subsubsection{Coke}

Total Quantity Used $=18445$ Tons

Moisture Content $=221$ Tons $=1.2 \%=4.57 \mathrm{Kg} / \mathrm{THM}=254 \mathrm{moles} / \mathrm{THM}$

Dry Weight $=18224$ Tons

The coke and coke ash composition analysis results are shown in Table 7 and Table 8 respectively.

Table 7. Coke Composition Analysis.

\begin{tabular}{cccc}
\hline Substance & Carbon & Ash & Inherent $\mathbf{H}_{\mathbf{2}} \mathbf{O}$ \\
\hline$\%$ & 86.7 & 12.35 & 0.95 \\
Quantity (Tons) & $15800=27198$ moles/THM & 2251 & $173=199 \mathrm{moles} / \mathrm{THM}$ \\
\hline
\end{tabular}

Table 8. Coke Ash Composition Analysis.

\begin{tabular}{cccccccc}
\hline Element & $\mathbf{S i O}_{2}$ & $\mathbf{F e}_{2} \mathbf{O}_{3}$ & $\mathbf{C a O}$ & $\mathbf{M g O}$ & $\mathbf{M n O}$ & $\mathbf{T i O}_{2}$ & $\mathbf{P}_{2} \mathbf{O}_{5}$ \\
\hline$\%$ & 53.54 & 10.21 & 5.8 & 1.76 & 0.23 & 1.48 & 1.37 \\
$\mathrm{Kg} / \mathrm{THM}$ & 24.9 & 4.8 & 2.68 & 1.62 & 0.11 & 0.69 & 0.64 \\
Moles/THM & 415 & 30 & 48 & 40.46 & 1.5 & 8.6 & 4.5 \\
\hline
\end{tabular}

\subsubsection{Coal}

Total Quantity Used $=6301$ Tons

Moisture Content $=8 \%=504$ Tons $=10.41 \mathrm{Kg} / \mathrm{THM}=578.38 \mathrm{moles} / \mathrm{THM}$

The coal and coal ash composition analysis results are shown in Table 9 and Table 10 respectively.

Table 9. Coal Composition Analysis.

\begin{tabular}{ccccc}
\hline Substance & Carbon & Volatile Matter & Ash & Inherent $\mathbf{H}_{2} \mathbf{O}$ \\
\hline$\%$ & 69.22 & 19.47 & 10.11 & 1.2 \\
Quantity (Tons) & $4361.55=7507.85 \mathrm{moles} / \mathrm{THM}$ & $1227=25.34 \mathrm{Kg} / \mathrm{THM}$ & 637 & $75.61=86.77 \mathrm{moles} / \mathrm{THM}$ \\
\hline
\end{tabular}


Table 10. Coal Ash Composition Analysis.

\begin{tabular}{|c|c|c|c|c|c|c|c|c|}
\hline Element & $\mathrm{SiO}_{2}$ & $\mathrm{Fe}_{2} \mathrm{O}_{3}$ & $\mathrm{CaO}$ & MgO & MnO & $\mathrm{TiO}_{2}$ & $\mathbf{P}_{2} \mathbf{O}_{5}$ & $\mathbf{A l}_{2} \mathbf{O}_{3}$ \\
\hline$\%$ & 43.04 & 6.69 & 5.96 & 1.31 & 0.04 & 1.28 & 1.17 & 35.89 \\
\hline $\mathrm{Kg} / \mathrm{THM}$ & 48.22 & 8.71 & 7.76 & 2.5 & 0.05 & 1.66 & 0.87 & 8.8 \\
\hline Moles/THM & 803.65 & 54.42 & 138.52 & 62.63 & 0.73 & 20.82 & 6.12 & 86.29 \\
\hline
\end{tabular}

\subsubsection{Nut Coke}

Total Quantity Used $=985$ Tons

Moisture Content $=8 \%=83$ Tons $=1.71 \mathrm{Kg} / \mathrm{THM}=95.24 \mathrm{moles} / \mathrm{THM}$

Dry Weight $=902$ Tons

The nut coke and nut coke ash composition analysis results are shown in Table 11 and Table 12 respectively.

Table 11. Nut Coke Composition Analysis.

\begin{tabular}{cccc}
\hline Substance & Carbon & Ash & \\
\hline$\%$ & 86.7 & 12.35 & Inherent $\mathbf{H}_{\mathbf{2}} \mathbf{O}$ \\
Quantity (Tons) & $782=1346.11 \mathrm{moles} / \mathrm{THM}$ & $111.4=2.3 \mathrm{Kg} / \mathrm{THM}$ & 0.95 \\
\hline
\end{tabular}

Table 12. Nut Coke Ash Composition Analysis.

\begin{tabular}{|c|c|c|c|c|c|c|c|c|}
\hline Element & $\mathrm{SiO}_{2}$ & $\mathrm{Fe}_{2} \mathrm{O}_{3}$ & $\mathrm{CaO}$ & MgO & MnO & $\mathrm{TiO}_{2}$ & $\mathbf{P}_{2} \mathbf{O}_{5}$ & $\mathrm{Al}_{2} \mathrm{O}_{3}$ \\
\hline$\%$ & 53.54 & 10.21 & 5.8 & 1.76 & 0.23 & 1.48 & 1.37 & 23.13 \\
\hline $\mathrm{Kg} / \mathrm{THM}$ & 1.83 & 0.23 & 0.13 & 0.04 & 0.05 & 0.03 & 0.03 & 2.36 \\
\hline Moles/THM & 30.46 & 1.47 & 2.38 & 1.01 & 0.7 & 0.42 & 0.22 & 5.21 \\
\hline
\end{tabular}

\subsection{Hot Metal Analysis}

Temperature of Hot Metal $=1464^{\circ} \mathrm{C}=1737 \mathrm{~K}$

The hot metal composition analysis results is shown in Table 13.

Table 13. Hot Metal Composition Analysis.

\begin{tabular}{cccccc}
\hline Element & Si & Mn & P & Ti & C \\
\hline$\%$ & 0.61 & 0.42 & 0.162 & 0.065 & 4.42 \\
Kg/THM & 6.1 & 4.2 & 1.62 & 0.65 & 44.2 \\
Moles/THM & 218 & 76.36 & 52.26 & 13.54 & 3684 \\
\hline
\end{tabular}

\subsubsection{Silicon}

Quantity of Si in Hot Metal= 218 moles/THM

Sensible Heat of Si in Hot Metal $(\mathrm{C} 1)=218 \mathrm{moles} / \mathrm{THM} * 21 \mathrm{Kcal} / \mathrm{moles}$

$$
=4578 \mathrm{Kcal} / \mathrm{moles}
$$

Heat of Solution of $\mathrm{Si}(\mathrm{C} 2)=218 \mathrm{moles} / \mathrm{THM} *-28.5 \mathrm{Kcal} / \mathrm{mole}$

$$
=-6213 \mathrm{Kcal} / \mathrm{mole}
$$

\subsubsection{Manganese}

Quantity of Mn in Hot Metal $=76.36$ moles $/$ THM

Sensible Heat of Mn in Hot Metal (C3) $=76.36 \mathrm{moles} / \mathrm{THM} * 17.89 \mathrm{Kcal} / \mathrm{mole}$

$$
=1366.08 \mathrm{Kcal} / \mathrm{THM}
$$

Heat of Solution of Mn $(\mathrm{C} 4)=76.36 \mathrm{moles} / \mathrm{THM} * 1.2 \mathrm{Kcal} / \mathrm{mole}$

$$
=91.63 \mathrm{Kcal} / \mathrm{THM}
$$

\subsubsection{Phosphorus}

Quantity of $\mathrm{P}$ in Hot Metal $=52.26 \mathrm{moles} / \mathrm{THM}$

$$
=26.13 \text { moles } / \text { THM }\left\{2 \mathrm{P} \rightarrow \mathrm{P}_{2}\right\}
$$

Sensible Heat of $\mathrm{P}$ in Hot Metal $(\mathrm{C} 5)=26.13 \mathrm{moles} / \mathrm{THM} * 46.266 \mathrm{Kcal} / \mathrm{mole}$

$$
=1209 \mathrm{Kcal} / \mathrm{THM}
$$

Heat of Solution of P (C6) $=26.13 \mathrm{moles} / \mathrm{THM} *-29.2 \mathrm{Kcal} / \mathrm{mole}$

$$
=-763 \mathrm{Kcal} / \mathrm{mole}
$$

\subsubsection{Titanium}

Quantity of Ti in Hot Metal $=13.54$ moles $/$ THM

Sensible Heat of Ti in Hot Metal (C7) $=13.54 \mathrm{moles} / \mathrm{THM} * 11.38 \mathrm{Kcal} / \mathrm{mole}$

$$
=154.08 \mathrm{Kcal} / \mathrm{THM}
$$


Heat of Solution of Ti $(\mathrm{C} 8)=13.54 \mathrm{moles} / \mathrm{THM} *-13.35 \mathrm{Kcal} / \mathrm{mole}$

$$
=-180.76 \mathrm{Kcal} / \mathrm{THM}
$$

\subsubsection{Carbon}

Quantity of C in Hot Metal $=3684$ moles $/$ THM

Sensible Heat of C in Hot Metal (C9) $=3684 \mathrm{moles} / \mathrm{THM} * 8.84 \mathrm{Kcal} / \mathrm{mole}$

$$
=32566.56 \mathrm{Kcal} / \mathrm{THM}
$$

Heat of Solution of C $(\mathrm{C} 10)=3684 \mathrm{moles} / \mathrm{THM} * 7.645 \mathrm{Kcal} / \mathrm{mole}$

$$
=28164.18 \mathrm{Kcal} / \mathrm{mole}
$$

The carbon entering into the hot metal, enters as elemental carbon. As it is considered all carbon to be undergoing oxidation in the furnace, the reversible reaction is taken to indicate the release back of elemental carbon.

$$
\mathrm{CO}_{2} \rightarrow \mathrm{C}+\mathrm{O}_{2} \quad \Delta \mathrm{H}_{\text {Reaction }}=94.14 \mathrm{Kcal} / \mathrm{mole}
$$

Latent Heat of Elemental Carbon $(\mathrm{C} 11)=3684 \mathrm{moles} / \mathrm{THM} * 94.14 \mathrm{Kcal} / \mathrm{mole}$

$$
=346811.76 \mathrm{Kcal} / \mathrm{mole}
$$

\subsubsection{Iron}

Quantity of Fe in Hot Metal $=941.752 \mathrm{Kg} / \mathrm{THM}$

$$
=16817 \text { moles } / \mathrm{THM}
$$

Sensible Heat of Fe in Hot Metal $(\mathrm{C} 12)=16817 \mathrm{moles} / \mathrm{THM} * 17.171 \mathrm{Kcal} / \mathrm{mole}$

$$
=288764.71 \mathrm{Kcal} / \mathrm{THM}
$$

Heat of Solution of $\mathrm{Fe}=16817 \mathrm{moles} / \mathrm{THM} * 0 \mathrm{Cal} / \mathrm{mole}$

$$
=0
$$

Total Heat of all elements in Hot Metal $=\mathrm{C} 1+\mathrm{C} 2+\mathrm{C} 3+\mathrm{C} 4+\mathrm{C} 5+\mathrm{C} 6+\mathrm{C} 7+\mathrm{C} 8+\mathrm{C} 9+\mathrm{C} 10+\mathrm{C} 11+\mathrm{C} 12$

$$
=696730 \mathrm{Kcal} / \mathrm{THM}(+)
$$

\subsection{Volatile Matter}

Assuming all volatile matter to be phenol. [4]

Heat Capacity of Phenol $=122 \mathrm{Kcal} / \mathrm{Kg}$

Heat of vaporization of coal volatile matter $=25.34 \mathrm{Kg} / \mathrm{THM} * 122 \mathrm{Kcal} / \mathrm{Kg}$

$$
=3091.48 \mathrm{Kcal} / \mathrm{THM}(+)
$$

\subsection{Moisture in Input Materials}

Total Moisture content in all input materials $=1662 \mathrm{moles} / \mathrm{THM}$

$$
=30 \mathrm{Kg} / \mathrm{THM}
$$

Sensible Heat absorbed by moisture $(\mathrm{E} 1)=1662 \mathrm{moles} / \mathrm{THM} * 1.353 \mathrm{Kcal} / \mathrm{mole}$

Latent Heat of Evaporation of Water $=540 \mathrm{Kcal} / \mathrm{Kg}$

$$
=2249 \mathrm{Kcal} / \mathrm{THM}
$$

\begin{tabular}{|c|c|c|c|c|c|}
\hline Element & $\mathrm{CO}$ & $\mathrm{CO}_{2}$ & $\mathrm{O}_{2}$ & $\mathbf{N}_{2}$ & $\mathrm{H}_{2}$ \\
\hline$\%$ & 23.74 & 20.09 & 0.71 & 53.41 & 2.05 \\
\hline $\mathrm{m}^{3} / \mathrm{THM}$ & 404 & 342 & 12 & 909 & 35 \\
\hline Moles/THM & 18024 & 15257 & 536 & 40580 & 1564 \\
\hline
\end{tabular}

Heat Lost in evaporation of moisture $(\mathrm{E} 2)=30 \mathrm{Kg} / \mathrm{THM} * 540 \mathrm{Kcal} / \mathrm{Kg}$

$$
=16200 \mathrm{Kcal} / \mathrm{THM}
$$

Total Heat Lost in evaporation of moisture $=\mathrm{E} 1+\mathrm{E} 2$

$$
=18449 \mathrm{Kcal} / \mathrm{THM}(+)
$$

\subsection{Blast Furnace Gas (BFG)}

Quantity of BFG $=1702 \mathrm{~m}^{3} / \mathrm{THM}$

Calorific Value of $\mathrm{BFG}=870 \mathrm{Kcal} / \mathrm{m}^{3}$

Temperature of Blast Furnace Gas $=179^{\circ} \mathrm{C}=452 \mathrm{~K}$

The blast furnace gas composition analysis results is shown in Table 14 .

Table 14. Blast Furnace Gas Composition Analysis.

Latent Heat of BFG $=$ Quantity * Calorific Value

$$
\begin{aligned}
& =1702 \mathrm{Nm}^{3} / \mathrm{THM} * 870 \mathrm{Kcal} / \mathrm{Nm}^{3} \\
& =1480740 \mathrm{Kcal} / \mathrm{THM}(+)
\end{aligned}
$$




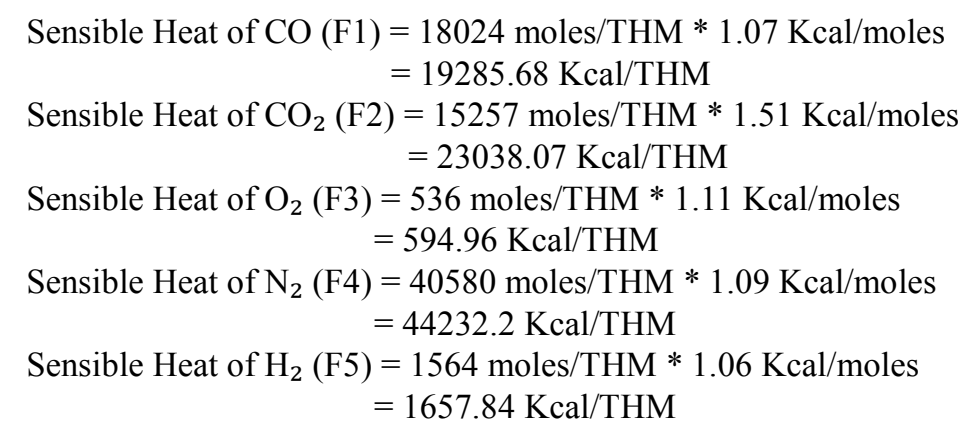

Total Sensible Heat of all elements in $\mathrm{BFG}=\mathrm{F} 1+\mathrm{F} 2+\mathrm{F} 3+\mathrm{F} 4+\mathrm{F} 5$

$=88809 \mathrm{Kcal} / \mathrm{THM}(+)$

\subsection{Dust Analysis}

Dust Formation Temperature $=1400 \mathrm{~K}$

The dust formation temperature is taken as $1400 \mathrm{~K}$ as the reactants with the dust, undergo reaction only after reaching the desired temperature. Also, dust is produced after a reaction, hence the calculation for sensible heat of dust will take into consideration the temperature in which a reaction takes place. Other than the mentioned compounds, dust also contains nominal amounts of $\mathrm{MnO}, \mathrm{TiO}_{2}, \mathrm{P}_{2} \mathrm{O}_{5}$. The heat effects of these compounds can be neglected. Table 15 shows the composition analysis results for the dust collected in dust catcher, and Table 16 shows the composition dust analysis results for the dust collected in gas cleaning plant.

\subsubsection{Dust Catcher}

Quantity of Dust Collected in Dust Catcher $=728$ Tons

Table 15. Composition of Dust Collected in Dust Catcher.

\begin{tabular}{|c|c|c|c|c|c|c|}
\hline Element & C & $\mathrm{Fe}_{2} \mathrm{O}_{3}$ & $\mathrm{SiO}_{2}$ & $\mathrm{Al}_{2} \mathrm{O}_{3}$ & $\mathrm{CaO}$ & MgO \\
\hline$\%$ & 12.1 & 68.24 & 6.84 & 5.84 & 2.55 & 1.37 \\
\hline $\mathrm{Kg} / \mathrm{THM}$ & 1.82 & 10.25 & 1.04 & 0.88 & 0.38 & 0.23 \\
\hline Moles/THM & 151.65 & 64.12 & 17.29 & 8.55 & 6.71 & 5.72 \\
\hline
\end{tabular}

Sensible Heat of C $(\mathrm{G} 1)=151.65 \mathrm{moles} / \mathrm{THM} * 4.99 \mathrm{Kcal} / \mathrm{mole}$ $=757 \mathrm{Kcal} / \mathrm{THM}$

Sensible Heat of $\mathrm{Fe}_{2} \mathrm{O}_{3}(\mathrm{G} 2)=64.12 \mathrm{moles} / \mathrm{THM} * 37.65 \mathrm{Kcal} / \mathrm{mole}$ $=2414 \mathrm{Kcal} / \mathrm{THM}$

Sensible Heat of $\mathrm{SiO}_{2}(\mathrm{G} 3)=17.29 \mathrm{moles} / \mathrm{THM} * 17.64 \mathrm{Kcal} / \mathrm{mole}$ $=305 \mathrm{Kcal} / \mathrm{THM}$

Sensible Heat of $\mathrm{Al}_{2} \mathrm{O}_{3}(\mathrm{G} 4)=8.55 \mathrm{moles} / \mathrm{THM} * 30.8 \mathrm{Kcal} / \mathrm{mole}$ $=263 \mathrm{Kcal} / \mathrm{THM}$

Sensible Heat of $\mathrm{CaO}(\mathrm{G} 5)=6.71 \mathrm{moles} / \mathrm{THM} * 13.43 \mathrm{Kcal} / \mathrm{mole}$ $=90 \mathrm{Kcal} / \mathrm{THM}$

Sensible Heat of $\mathrm{MgO}(\mathrm{G} 6)=5.72 \mathrm{moles} / \mathrm{THM} * 12.57 \mathrm{Kcal} / \mathrm{mole}$ $=72 \mathrm{Kcal} / \mathrm{THM}$

Total Sensible Heat of Dust Collected in Dust Catcher (P8) = G1 + G2 + G3 + G4 + G5 + G6

\subsubsection{Gas Cleaning Plant}

$=3901 \mathrm{Kcal} / \mathrm{THM}$

Quantity of Dust Collected $=478$ Tons

Table 16. Composition of Dust Collected in Gas Cleansing Plant (GCP).

\begin{tabular}{ccccccc}
\hline Element & $\mathbf{C}$ & $\mathbf{F e}_{2} \mathbf{O}_{3}$ & $\mathbf{S i O}_{2}$ & $\mathbf{A l}_{2} \mathbf{O}_{3}$ & $\mathbf{C a O}$ & $\mathbf{M g O}$ \\
\hline$\%$ & 23.48 & 52.66 & 7.62 & 6.03 & 2.5 & 2.22 \\
$\mathrm{Kg} / \mathrm{THM}$ & 2.32 & 5.2 & 0.75 & 0.95 & 0.25 & 0.22 \\
$\mathrm{Moles} / \mathrm{THM}$ & 193.2 & 32.5 & 12.51 & 5.92 & 4.41 & 5.48 \\
\hline
\end{tabular}

Sensible Heat of C (G7) $=193.2 \mathrm{moles} / \mathrm{THM} * 4.99 \mathrm{Kcal} / \mathrm{mole}$

$=964 \mathrm{Kcal} / \mathrm{THM}$

Sensible Heat of $\mathrm{Fe}_{2} \mathrm{O}_{3}(\mathrm{G} 8)=32.5 \mathrm{moles} / \mathrm{THM} * 37.65 \mathrm{Kcal} / \mathrm{mole}$

$$
=1224 \mathrm{Kcal} / \mathrm{THM}
$$




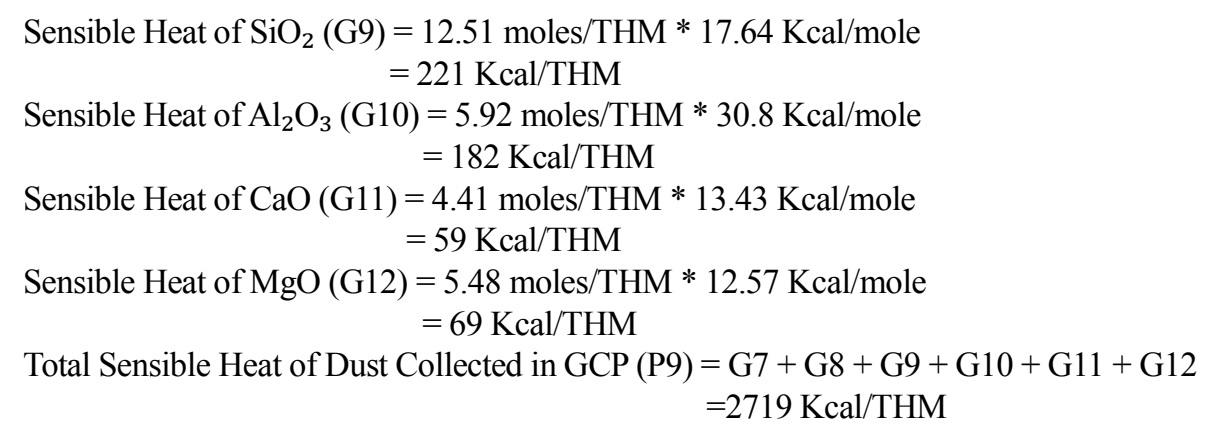

The dust comes out of the furnace in elemental form. As it is considered all carbon to be undergoing oxidation in the furnace, the reversible reaction is taken to indicate the release back of elemental carbon.

$$
\mathrm{CO}_{2}(\mathrm{~g}) \rightarrow \mathrm{C}(\mathrm{s})+\mathrm{O}_{2}(\mathrm{~g}) \quad \Delta \mathrm{H}_{\text {Reaction }}=94.14 \mathrm{Kcal} / \mathrm{mole}
$$

Latent Heat of Carbon Dust $(\mathrm{P} 10)=(151.65+193.2=344.85) \mathrm{moles} / \mathrm{THM} * 94.14 \mathrm{Kcal} / \mathrm{mole}$

$$
=32464 \mathrm{Kcal} / \mathrm{THM}(+)
$$

Total Heat in Dust $=\mathrm{P} 8+\mathrm{P} 9+\mathrm{P} 10$

$$
=39084 \mathrm{Kcal} / \mathrm{THM}(+)
$$

\subsection{Cooling by Water}

Quantity of Water Supplied=17014.3 Kg/THM

Rise in Temperature of water $=10^{\circ} \mathrm{C}$

Heat Capacity of Water $=1 \mathrm{Kcal} / \mathrm{Kg}^{\circ} \mathrm{C}$

Heat carried away by water $=$ Mass * Heat Capacity $*$ Temperature Difference $=170143 \mathrm{Kcal} / \mathrm{THM}(+)$

\subsection{Slag Analysis}

Quantity of Slag Produced $=362.2 \mathrm{Kg} / \mathrm{THM}$

\begin{tabular}{|c|c|c|c|c|c|c|c|}
\hline Element & $\mathrm{SiO}_{2}$ & $\mathrm{Al}_{2} \mathrm{O}_{3}$ & $\mathrm{FeO}$ & $\mathrm{CaO}$ & MgO & $\overline{\mathrm{TiO}_{2}}$ & MnO \\
\hline$\%$ & 33.36 & 18.57 & 0.73 & 34.53 & 9.32 & 0.91 & 0.83 \\
\hline $\mathrm{Kg} / \mathrm{THM}$ & 120.82 & 67.26 & 2.64 & 125.06 & 33.75 & 3.30 & 3 \\
\hline Moles/THM & 2014 & 659 & 37 & 2233 & 844 & 41 & 42 \\
\hline
\end{tabular}

The blast furnace slag composition analysis results is shown in Table 17.

Table 17. Slag Composition Analysis.

The slag calculation is done taking reference from [1]. The values of [1] are selected, as the slag composition data is similar to that produced in the blast furnace, taken in the study.

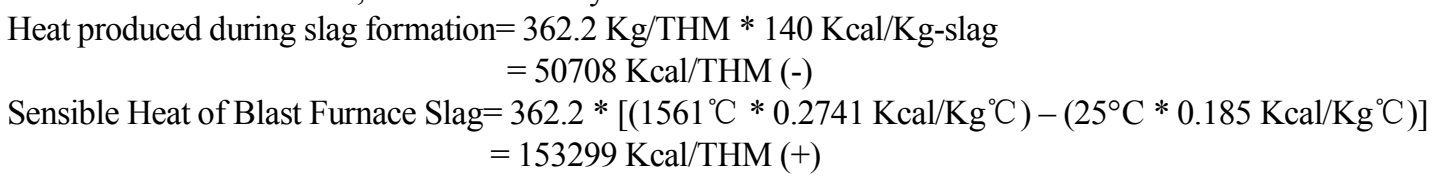

\subsection{Reactions}

Table 18 shows the elements entering into the blast furnace for reactions, after excluding dust losses, carbon in hot metal and FeO in slag. The mentioned elements have been excluded in beforehand for more accuracy in results. The amounts excluded are taken from

\begin{tabular}{|c|c|c|c|c|c|c|c|}
\hline Elements & $\mathrm{Fe}_{2} \mathrm{O}_{3}$ & $\mathrm{FeO}$ & $\mathrm{C}$ & $\mathrm{CaCO}_{3}$ & $\mathrm{CaO}$ & $\mathrm{MgCO}_{3}$ & MgO \\
\hline Moles/THM & 7624 & 1534 & 33611 & 31 & 2202 & 162 & 856 \\
\hline Elements & $\mathrm{SiO}_{2}$ & $\mathrm{MnO}$ & $\mathbf{P}_{2} \mathbf{O}_{5}$ & $\mathrm{TiO}_{2}$ & $\mathrm{H}_{2} \mathrm{O}$ & $\mathrm{O}_{2}$ & $\mathbf{A l}_{2} \mathbf{O}_{3}$ \\
\hline Moles/THM & 2232 & 118 & 31 & 67 & 2677 & 11607 & 659 \\
\hline
\end{tabular}
the respective composition sheets.

Table 18. Elements Entering the Blast Furnace for Reactions after Excluding Dust Losses, Carbon in Hot Metal and FeO in Slag.

The enthalpies of reactions, as shown in Table 19, are calculated according to the temperature in which the reaction takes place, by applying Kirchoff's equation (1).

$$
\left(\Delta \mathrm{H}_{\text {Reaction }}\right)_{\mathrm{T} 2}=\left(\Delta \mathrm{H}_{\text {Reaction }}\right)_{\mathrm{T} 1}+\left[\sum\left(\Delta \mathrm{H}_{\mathrm{T} 2}-\Delta \mathrm{H}_{\mathrm{T} 1}\right)_{\text {Products }}-\sum\left(\Delta \mathrm{H}_{\mathrm{T} 2}-\Delta \mathrm{H}_{\mathrm{T} 1}\right)_{\text {Reactants }}\right]
$$


where, $\mathrm{T} 2$ is the temperature at which the reaction takes place and $\mathrm{T} 1$ is the standard temperature of $298 \mathrm{~K}$. Figure 3 shows the different temperature zones in a blast furnace.

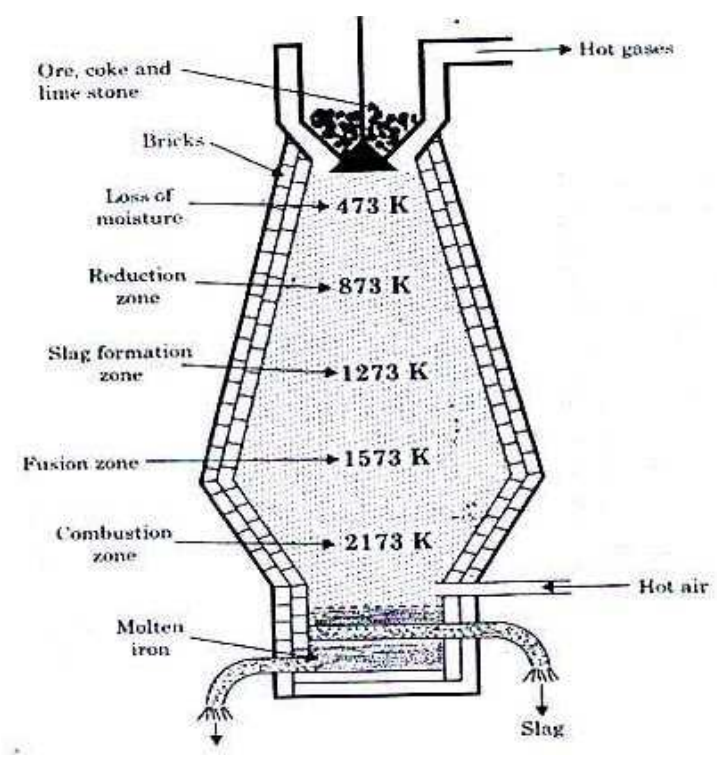

Figure 3. Temperature Zones in a Blast Furnace.

The $\mathrm{CO}$ produced, for the reduction of ores, is produced by the combustion of fuels at $1700 \mathrm{~K}$.

$$
2 \mathrm{C}(\mathrm{s})(23214)+\mathrm{O}_{2}(\mathrm{~g})(11607) \rightarrow 2 \mathrm{CO}(\mathrm{g})(23214)
$$

The values in brackets is the number of moles of the substance undergoing reaction according to the mass balance shown in Table 18.

After performing various calculations, the reduction percentages are taken to be $60 \%$ Indirect Type (CO) Reduction, $35 \%$ Direct Type (C) and 5\% reduction by Hydrogen $\left(\mathrm{H}_{2}\right)$, as this yields the most accurate results, as shown in Table 19. These percentages vary according to the operating conditions of the furnace. The percentages can be estimated by taking into consideration the BF Gas analysis.

Table 19. Blast Furnace Reactions and Enthalpy.

\begin{tabular}{|c|c|c|c|}
\hline Reactions (Value in brackets indicate number of moles) & Temperature (K) & $\Delta \mathbf{H}_{\text {Reaction }}(\mathrm{Kcal} / \mathrm{mole})$ & Total $\Delta \mathbf{H}($ Kcal) \\
\hline $\mathrm{C}(\mathrm{s})(2677)+\mathrm{H}_{2} \mathrm{O}(\mathrm{g})(2677) \rightarrow \mathrm{CO}(\mathrm{g})(2677)+\mathrm{H}_{2}(\mathrm{~g})(2677)$ & 1700 & +32.1 & +85931.7 \\
\hline $3 \mathrm{Fe}_{2} \mathrm{O}_{3}(\mathrm{~s})(4574)+\mathrm{CO}(\mathrm{g})(1525) \rightarrow 2 \mathrm{Fe}_{3} \mathrm{O}_{4}(\mathrm{~s})(3049)+\mathrm{CO}_{2}(\mathrm{~g})(1525)$ & 1000 & -12.47 & -19012.59 \\
\hline $\mathrm{Fe}_{3} \mathrm{O}_{4}(\mathrm{~s})(3049)+\mathrm{CO}(\mathrm{g})(3049) \rightarrow 3 \mathrm{FeO}(\mathrm{s})(9147)+\mathrm{CO}_{2}(\mathrm{~g})(3049)$ & 1200 & +4.585 & +13979.66 \\
\hline $\mathrm{FeO}(\mathrm{s})(9147+921=10068)+\mathrm{CO}(\mathrm{g})(10068) \rightarrow \mathrm{Fe}(\mathrm{s})(10068)+\mathrm{CO}_{2}(\mathrm{~g})(10068)$ & 1200 & -3.85 & -38761.8 \\
\hline $3 \mathrm{Fe}_{2} \mathrm{O}_{3}(\mathrm{~s})(2668)+\mathrm{C}(\mathrm{s})(889) \rightarrow 2 \mathrm{Fe}_{3} \mathrm{O}_{4}(\mathrm{~s})(1179)+\mathrm{CO}(\mathrm{g})(889)$ & 1400 & +26.95 & +23967.53 \\
\hline $\mathrm{Fe}_{3} \mathrm{O}_{4}(\mathrm{~s})(1179)+\mathrm{C}(\mathrm{s})(1179) \rightarrow 3 \mathrm{FeO}(\mathrm{s})(3537)+\mathrm{CO}(\mathrm{g})(1179)$ & 1400 & +44.43 & +52383 \\
\hline $\mathrm{FeO}(\mathrm{s})(3537+537=4074)+\mathrm{C}(\mathrm{s})(4074) \rightarrow \mathrm{Fe}(\mathrm{s})(4074)+\mathrm{CO}(\mathrm{g})(4074)$ & 1700 & +27.48 & +111953.5 \\
\hline $3 \mathrm{Fe}_{2} \mathrm{O}_{3}(\mathrm{~s})(381)+\mathrm{H}_{2}(\mathrm{~g})(127) \rightarrow 2 \mathrm{Fe}_{3} \mathrm{O}_{4}(\mathrm{~s})(254)+\mathrm{H}_{2} \mathrm{O}(\mathrm{g})(127)$ & 1200 & -46 & -5842 \\
\hline $\mathrm{Fe}_{3} \mathrm{O}_{4}(\mathrm{~s})(254)+\mathrm{H}_{2}(\mathrm{~g})(254) \rightarrow 3 \mathrm{FeO}(\mathrm{s})(762)+\mathrm{H}_{2} \mathrm{O}(\mathrm{g})(254)$ & 1200 & +12.45 & +3162.3 \\
\hline $\mathrm{FeO}(\mathrm{s})(762+76=838)+\mathrm{H}_{2}(\mathrm{~g})(838) \rightarrow \mathrm{Fe}(\mathrm{s})(838)+\mathrm{H}_{2} \mathrm{O}(\mathrm{g})(838)$ & 1300 & +3.73 & +3126 \\
\hline $\mathrm{CaCO}_{3}(\mathrm{~s})(31) \rightarrow \mathrm{CaO}(\mathrm{s})(31)+\mathrm{CO}_{2}(\mathrm{~g})(31)$ & 1200 & +39.42 & +1222.02 \\
\hline $\mathrm{MgCO}_{3}(\mathrm{~s})(162) \rightarrow \mathrm{MgO}(\mathrm{s})(162)+\mathrm{CO}_{2}(\mathrm{~g})(162)$ & 700 & +27 & +4374 \\
\hline $\mathrm{P}_{2} \mathrm{O}_{5}(\mathrm{~s})(26)+5 \mathrm{C}(\mathrm{s})(130) \rightarrow 2 \mathrm{P}(\mathrm{s})(52)+5 \mathrm{CO}(\mathrm{g})(130)$ & 1700 & +206 & +5356 \\
\hline $\mathrm{TiO}_{2}(\mathrm{~s})(13)+2 \mathrm{C}(\mathrm{s})(26) \rightarrow \mathrm{Ti}(\mathrm{s})(13)+2 \mathrm{CO}(\mathrm{g})(26)$ & 1700 & +129.36 & +1681.68 \\
\hline $\mathrm{MnO}(\mathrm{s})(76)+\mathrm{C}(\mathrm{s})(76) \rightarrow \mathrm{Mn}(\mathrm{s})(76)+\mathrm{CO}(\mathrm{g})(76)$ & 1700 & +69.33 & +5269.08 \\
\hline $\mathrm{SiO}_{2}(\mathrm{~s})(218)+2 \mathrm{C}(\mathrm{s})(436) \rightarrow \mathrm{Si}(\mathrm{s})(218)+2 \mathrm{CO}(\mathrm{g})(436)$ & 1700 & +158.89 & +34638.02 \\
\hline $\mathrm{CO}(\mathrm{g})(1219)+\mathrm{H}_{2} \mathrm{O}(\mathrm{g})(1219) \rightarrow \mathrm{CO}_{2}(\mathrm{~g})(1219)+\mathrm{H}_{2}(\mathrm{~g})$ & 1000 & -8.33 & -20350.19 \\
\hline $\mathrm{C}(\mathrm{s})(910)+\mathrm{CO}_{2}(\mathrm{~g})(910) \rightarrow 2 \mathrm{CO}(\mathrm{s})(1820)$ & 1300 & +40.05 & +36445.5 \\
\hline $\mathrm{Fe}(\mathrm{s})(16780) \rightarrow \mathrm{Fe}(1)(16780)$ & 1800 & +3.56 & +59737 \\
\hline$\sum$ Summation of Enthalpies & & & +359261 \\
\hline
\end{tabular}

Reaction $\mathrm{X}$ is the mass balancing equation to balance the excess C supplied (excess fuel).
Moles produced in the $\mathrm{BF}$ according to calculations: $\mathrm{CO}=18660 ; \mathrm{CO}_{2}=15144$ 
But the moles leaving the furnace in BF Gas:

$\mathrm{CO}=18024 ; \mathrm{CO}_{2}=15257$

Difference in moles of $\mathrm{CO}=18660-18024=636$ (Excess)

Difference in moles of $\mathrm{CO}_{2}=15144-15257=113$ (Less)

This difference in the number of moles can be explained due to the other reactions taking place inside the furnace. It can be said that 113 moles of $\mathrm{CO}$ are reducing some other oxides (other than those taken in this study) to produce 113 moles of $\mathrm{CO}_{2}$. Hence $\mathrm{CO}_{2}$ balance will then be achieved. Excess CO (523) may be due to the following reasons: 1 . Furnace having a lesser percentage of direct reduction $(<35 \%)$. 2. Excess fuel supply (From reaction $X$ )

Reaction $\mathrm{X}$ is indicative to the excess fuel supplied inside the furnace. Though some amount of excessive fuel is desirable in order, to maintain the working temperature of the furnace. High amount of excessive fuel would produce negative effects on the efficiency of the furnace. As from above, reaction $\mathrm{X}$, is an endothermic reaction, so adding extra fuel will reduce the efficiency of the furnace and also disturb the $\mathrm{CO} / \mathrm{CO}_{2}$ gas balance, which is undesirable. In general, around $8-10 \mathrm{Kgs}$ of fuel is supplied in excess to prevent cooling down of the furnace. However, any quantity more than this, is wastage of fuel and should be restricted.

\section{Heat Energy Balance Sheet}

The final heat energy balance sheet is shown in Table 20 .

Table 20. Heat Energy Balance Sheet.

\begin{tabular}{lcc}
\hline HEAT INPUTS & & \\
\hline Sources & $\mathbf{\Delta H}$ (Kcal/THM) & $\mathbf{\%}$ \\
\hline Energy from Fuel & 3533420 & 85.90 \\
Energy from Combustion Air & 528378 & 12.85 \\
Energy from Slag Production & 50708 & 1.25 \\
Total Heat Input & $\mathbf{4 1 1 2 5 0 6}$ & $\mathbf{1 0 0}$ \\
\hline HEAT OUTPUTS & & \\
\hline Sources & $\mathbf{\Delta H}$ (Kcal/THM) & $\mathbf{\%}$ \\
\hline Sensible Heat of Hot Metal & 696730 & 16.94 \\
Sensible Heat in Volatile Matter & 3091 & 0.07 \\
Heat needed to vaporize Moisture & 18929 & 0.46 \\
Latent Heat in BF Gas & 1480740 & 36.02 \\
Sensible Heat in BF Gas & 88809 & 2.15 \\
Total Heat in Dust (Dust Catcher + GCP) & 39084 & 0.95 \\
Heat Carried away by Water & 170143 & 4.14 \\
Sensible Heat in Slag & 153299 & 3.73 \\
Summation of BF Reactions & 359261 & 8.73 \\
Calculated Heat Output & $\mathbf{3 0 1 0 0 8 6}$ & $\mathbf{7 3 . 1 9}$ \\
Other Heat Losses & $\mathbf{1 1 0 2 4 2 0}$ & $\mathbf{2 6 . 8 1}$ \\
Total Heat Output & $\mathbf{4 1 1 2 5 0 6}$ & $\mathbf{1 0 0}$ \\
\hline
\end{tabular}

\section{Conclusion}

A static heat energy balance mathematical model has been developed in this study. The model takes into consideration all the factors which play a significant role, in the heat requirements of a blast furnace. The calculations of the balance presented in the study will help to estimate the heat requirements of a furnace, and eradicate any excessive fuel supply. Individual percentage composition of each input and output component, taken in the study, would help to alter the quantity of supply of any component, for improving the efficiency. From the above calculations, one can account for approximately $73 \%$ of the heat supplied [Table 20]. This result is in accordance with the literature of heat energy balance for a blast furnace [3-5]. The remaining $27 \%$ can be explained as other heat losses taking place in the tuyere region [9-10], conduction, convection, radiation etc.

\section{References}

[1] Ertem, M. Emre, and Sabit Gürgen. "Energy balance analysis for Erdemir blast furnace number one." Applied thermal engineering 26.11 (2006): 1139-1148.

[2] Peacey, John G., and William George Davenport. The iron blast furnace: theory and practice. Elsevier, 2016.

[3] Habashi, Fathi. Principles of extractive metallurgy. Vol. 1. CRC Press, 1970.

[4] Sarangi, Arabinda, and BIDYAPATI SARANGI. Alternative Routes to Iron Making. PHI Learning Pvt. Ltd., 2016.

[5] Mustoe, L. H. "Principles of blast furnace ironmaking: Theory and practice: By AK Biswas; published by Cootha Publishing House, Brisbane, 1981.

[6] Kelly, K. K. "Heats of Fusion of Inorganic Compounds." US Bur. Mines Bull 393 (1936).

[7] Kelly, K. K. "High-temperature heat content, heat capacity, and entropy data for inorganic compounds." US Department of the Interior, Bureau of Mines Bulletin 476 (1949): 1-235.

[8] Elliott, John F., Molly Gleiser, and V. Ramakrishna. "Thermochemistry for steelmaking: thermodynamic and transport properties, v. 2." (1963).

[9] Kirillova, N. L., A. G. Radyuk, and A. E. Titlyanov. "Reducing heat loss through the surface of blast-furnace tuyeres." Metallurgist 57.9-10 (2014): 878-882.

[10] A. V. Borodulin, A. P. Vasil'ev, E. L. Glushchenko, et al., Proc. 2nd Int. Sci.-Tech. Conf. Automated Furnaces and Energy-Saving Technologies in Metallurgy, Dec. 3-5, 2002, Moscow, pp. 424-426.

[11] Agrawal, Ashish, et al. "A mathematical model to control thermal stability of blast furnace using proactive thermal indicator." Ironmaking \& Steelmaking (2017): 1-8.

[12] Hou, Qinfu, et al. "DEM-based virtual experimental blast furnace: A quasi-steady state model." Powder Technology 314 (2017): 557-566.

[13] Shen, Yansong, et al. "Modeling of Internal State and Performance of an Ironmaking Blast Furnace: Slot vs Sector Geometries." Metallurgical and Materials Transactions B 47.2 (2016): 1052-1062.

[14] David, Sayd Farage, Felipe Farage David, and M. L. P. Machado. "Artificial Neural Network Model for Predict of Silicon Content in Hot Metal Blast Furnace." Materials Science Forum. Vol. 869. Trans Tech Publications, 2016. 